\title{
Is 'minimally adequate treatment' really adequate? investigating the effect of mental health treatment on quality of life for children with mental health
}

\section{problems}

\section{Introduction}

Despite increased investment in prevention, screening, diagnosis and treatment for childhood mental health disorders in the last three decades, their prevalence remains stable at around $13 \%$ worldwide (Polanczyk et al., 2015; Sawyer et al., 2018b). One explanation for this apparent lack of improvement is inadequacy of treatment (Jorm et al., 2017). Half to three-quarters of children and adolescents with mental health disorders go untreated (Kataoka et al., 2002; Merikangas et al., 2011). Clinical trials in children show psychological and pharmacological therapies to be efficacious when delivered according to a strict protocol in defined populations, but in routine practice settings even 'treated' children may not receive effective treatment, as a result of deficiencies in the quantity and/or quality of treatment, both of which are largely unknown (Trask and Garland, 2012; Weisz and Jensen, 2001). Minimally adequate treatment (MAT) is a concept that arose in the adult mental health context (Wang et al., 2005), based on clinical practice guidelines regarding the minimum level of treatment considered sufficient to treat common mental health problems. To date, MAT and related metrics have largely been used to assess the distribution of mental health care (Hahm et al., 2015; Puyat et al., 2016; Thornicroft et al., 2017). Few studies have examined the relationship of MAT to outcomes, and existing studies are all among adults and often focus on adequacy of pharmacotherapy (Cully et al., 2008; Dewa et al., 2003; Smith et al., 2009). Those findings may be less relevant for children's treatment, where first line recommendations for many disorders are psychological rather than 
pharmacological. The limited available evidence from these studies highlights the importance of addressing selection bias when studying the impact of MAT, since patients receiving MAT are often those with more severe problems in observational studies without random allocation of treatment (Duhoux et al., 2013; Fortney et al., 2001). Child mental health researchers have used varying definitions of MAT to examine the distribution and equitability of mental health care. A recent study defined MAT for a range of children's mental health problems as eight or more mental health visits within twelve months, or four to seven mental health visits plus concurrent psychotropic medication (Sawyer et al., 2018a). This definition is consistent with treatment guidelines for common mental disorders in children, and was earlier used by Saloner et al. (2014) in the US. Sawyer et al. (2018a) showed that in Australia, only $11 \cdot 6 \%$ of a population sample of children with identified mental health disorders received this definition of MAT. In such studies, shortfalls in MAT are framed as inadequacies in health system performance, using criteria such as unmet need or inequalities of treatment. The importance of these findings is grounded on the implicit assumption that MAT improves outcomes. There is, however, no evidence as to whether MAT relates to outcomes in children with mental health problems. Quality of life is a key outcome measure to consider in child mental health functioning as it captures a broad view of functioning and impairments, including elements of potential treatment effect that may be missed by focusing only on symptoms as outcomes (Adamo et al., 2015). Quality of life captures both physical and psychosocial (including emotional and social) aspects of health and involves a subjective judgement of functioning, which for children is often provided by parents (Coghill et al., 2009). This paper aims to examine whether receipt of MAT by children with mental health symptoms is associated with better quality of life in a populationlevel dataset, to inform the use of MAT as a measure of health system performance. 


\section{Methods}

\section{$\underline{\text { Data }}$}

The Longitudinal Study of Australian Children (LSAC) (Gray and Sanson, 2005) is a nationally representative longitudinal cohort study involving face-to-face interviews with a parent (usually the mother) at approximately two-yearly intervals, plus self-completion questionnaires and links to external datasets including healthcare administrative data. This study uses data from the B cohort (recruited when the study child was 0-1 years old in 2004/05) and the K cohort (recruited when the study child was 4-5 years old in 2004/05), followed up to wave 7, when children from each cohort were aged 12-13 and 16-17, respectively.

At enrolment, $97 \%$ of families provided permission to link their survey data with administrative Medicare data. Medicare is the universal public health insurance scheme in Australia and primary funder of health care. It provides subsidies to all citizens and permanent residents for medical services under the Medicare Benefits Schedule (MBS) and for pharmaceutical prescriptions under the Pharmaceutical Benefits Scheme (PBS). Under this system, out-ofhospital services including visits to doctors and some allied health care professionals, diagnostic tests, and pharmaceutical prescriptions are subsidized. Patients may pay an out of pocket cost on top of the subsidy, but the linked MBS data includes all subsidized services. The MBS and PBS data list dates of claims for benefits classified under the two schemes. MBS data capture eligible visits to primary care physicians (called general practitioners or GPs in Australia) and specialists (including psychiatrists), plus psychologists and allied health professionals if the subsidy is claimed. PBS data record names of medications dispensed. No diagnostic information is recorded in these administrative data. Mental health-related care can be identified either by type 
of provider, such as psychiatrist or psychologist, or visits to GPs if the doctor claims a mental health-specific item number (such as mental health care plans or treatment visits). Mental health care that occurs without a specific mental health claim (for example as part of a visit to a GP combined with other care) or that is provided outside the public health insurance system (such as a privately funded visit to a psychologist) is not captured in MBS data.

The authors assert that all procedures contributing to this work comply with the ethical standards of the relevant national and institutional committees on human experimentation and with the Helsinki Declaration of 1975, as revised in 2008. All procedures involving human subjects/patients for LSAC were approved by the Australian Institute of Family Studies Ethics Committee and families consented to participate in writing. No specific ethics approval was required for this study because it used secondary deidentified data made available by the LSAC administrators.

\section{$\underline{\text { Participants/sample }}$}

The Strengths and Difficulties Questionnaire (SDQ) is a widely used and validated measure of child psychopathology in population samples (Goodman et al., 2000). It has five subscales (each comprising five items): emotional problems, peer relationships, conduct problems, hyperactivity, and prosocial behavior. These can be combined to form the internalizing (peer and emotional) and externalizing (conduct and hyperactivity) problem scores (Goodman et al., 2010). A total score of 17 or more indicates a high level of mental health symptoms; approximately $10 \%$ of the general child population score above this cut-point (Mellor, 2005; Youth in Mind, 2016). This measure has excellent correlation with child mental health as assessed via diagnostic interview 
(Hawes and Dadds, 2004). This study used parent-reported SDQ; child-reported and teacherreported SDQ were not used to define the main sample due to high levels of missing data.

The sample was defined as LSAC study children with SDQ of 17 or more at wave 5 or 6 of the survey (ages 8-15). 'Baseline' is defined as the interview date of the first of these two waves at which SDQ score was 17 or more. The interview date of the subsequent wave (ages 10-17) is termed 'follow-up' as shown in Figure 1. For the 751 children in LSAC who had SDQ of 17 or more at wave 5 or 6,688 had the required four periods of observation; of these 598 had the outcome measure at all four periods; and of these 2 had missing covariate data. The final sample of 596 children comprised $9 \cdot 9 \%$ of all 6,028 children with outcome data available (Youth in Mind, 2016). SDQ data were missing for $1.6 \%$ of the children in LSAC at wave 5, and $2.8 \%$ at wave 6.

Figure 1. Overview of measures used in the study

[Insert Figure 1 here]

$\mathrm{W}=$ wave, $\mathrm{SDQ}=$ strengths and difficulties questionnaire, $\mathrm{t}-1=$ one wave prior to baseline, $\mathrm{t}-2=$ two waves prior to baseline

\section{$\underline{\text { Measures }}$}

Primary outcome measure

Quality of life was measured by the 23-item parent-reported Paediatric Quality of Life Inventory (PEDSQL), a validated measure of children's health related quality of life in population samples 
(Varni et al., 2003). It consists of psychosocial and physical subscales, with the psychosocial subscale made up of three further subscales: emotional, social, and school. Each item is rated based on the child's functioning over the prior six months on a four-point scale, from never/rarely (0) to very often (3).

\section{MAT treatment variable}

We constructed variables capturing mental health service use and medication prescriptions between baseline and follow-up using the linked MBS and PBS data. Prior to 1 April 2012, PBS data did not capture all prescriptions, so we limited our sample to examine treatment after this date to avoid missing prescriptions.

Following the definition of MAT from Sawyer et al. (2018a) and Saloner et al. (2014) that was based on treatment guidelines, MAT was defined over a twelve-month period and comprised either 8 or more mental health visits, or 4 or more mental health visits plus relevant medication. Eligible visits were: mental health visits to a GP, social worker, or occupational therapist, or any visit to a psychiatrist, psychologist, or pediatrician. In Australia, pediatricians are considered specialists rather than primary care providers, and GPs are the providers of primary physician care. The medications covered by this concept of MAT were: amitriptyline, atomoxetine, aripiprazole, carbamazepine, chlorpromazine, citalopram, clomipramine, clonazepam, clonidine, desvenlafaxine, dexamphetamine, diazepam, duloxetine, escitalopram, fluoxetine, fluvoxamine, imipramine, lamotrigine, lithium, methylphenidate, mirtazapine, nitrazepam, olanzapine, paroxetine, quetiapine, risperidone, sertraline, temazepam, topiramate, valproate, and venlafaxine. Mental health visits to the family practitioner were defined according to the Medicare Benefits Schedule (MBS) item numbers 2700/2701/2712/2713/2715/2717/2725. Three 
categories of treatment were constructed: 'no treatment' (no mental health visit or medication), 'some treatment' (at least one visit or medication but not qualifying as MAT), and 'MAT'.

\section{Covariates}

The analysis accounted for other factors that might influence children's outcomes and/or their utilization of healthcare. Details of these other factors are provided in supplementary material (TableS1). This included factors affecting accessibility of healthcare, including household income and remoteness, an important factor given Australia's geography with those in remote areas having much lower access to mental health services. We also accounted for other factors influencing families' utilization of healthcare for the child: parental mental health, ethnicity, language, and maternal education. From survey data at baseline, we identified whether the parent had reported a diagnosed mental health problem for their child, and from healthcare administrative data prior to baseline we captured whether the child had previously received any mental health treatment, both of which might influence the likelihood of subsequent access to treatment.

\section{$\underline{\text { Statistical approach }}$}

The analysis examined the effect of treatment between baseline and follow-up on children's quality of life at follow-up, accounting for baseline quality of life. Treatment categories of 'MAT' and 'No treatment' were compared to the reference category of 'Some treatment' since we wanted to identify the impact of the minimally adequate level of treatment compared to lower levels, rather than the effect of any treatment compared to none. 
Due to the observational nature of the data, treatment was not randomly assigned. Differences in baseline characteristics between treatment categories were therefore tested using one-way analysis of variance for continuous variables and chi-square tests for binary variables. A priori we hypothesized that MAT would be more likely among those with lower quality of life, since previous research has shown that MAT is more common among those higher severity of mental health problems (Sawyer et al., 2018a). We also hypothesized that unobserved factors such as type of mental health problem and attitudes to help-seeking could confound the association between treatment and outcomes. To address this, we applied the lagged dependent variable (LDV) regression modelling approach, which allows quality of life for the treated and comparison groups to follow different patterns prior to the treatment period. It includes the outcome measure at and prior to baseline in the model, capturing time-varying unobserved factors which confound the relationship between treatment and outcome. O'Neill et al. (2016) compared LDV to other approaches that allow for pre-existing differential trends prior to treatment, and found that LDV was more efficient with lower bias than synthetic control and matching approaches. All analyses were conducted in Stata (version 15·0, Texas, USA).

\section{Robustness checks}

To assess the robustness of our results to alternative model specifications and assumptions, we tested:

1. Whether treatment improved mental health, represented by the SDQ internalizing and externalizing problem scores, as an intermediate step to improved quality of life.

2. The association between MAT and each of the subscales of the psychosocial scale of the PEDSQL - emotional, social, and school subscales. 
3. An alternate definition of MAT: GPs were financially incentivized to conduct mental health plans, through which patients could access up to six psychological therapy sessions subsidized by public health insurance before review by the GP (MBS Online). We defined 'implemented GP mental health plans' as a mental health plan plus at least six eligible mental health visits. Because this definition of MAT did not include medication, we could include events prior to 1 April 2012, and included in the sample all children with SDQ of $\geq 17$ at waves 3 to 6 of LSAC.

4. Unlike for GPs, there is no mental health identifier for visits to pediatricians. The main analysis included all pediatrician visits in determining MAT; as an alternative, all pediatrician visits were dropped from the definition of MAT.

5. To reduce the likelihood of including children without mental health problems in the sample we varied the inclusion criterion to SDQ scores of 20 or more at wave 5 or 6. Approximately 5\% of children would be expected to score above this higher threshold (Youth in Mind, 2016).

6. Self-reported SDQ was available for later waves of LSAC, starting when children were aged 10-11 years. Parent-reported SDQ is more reliable for detecting mental disorders, but self-report can be useful for detection of emotional problems (Goodman et al., 2000). We defined the sample using self-report as an alternative to parent-reported SDQ, but were not able to use it as an outcome measure since that would have required it to be included in earlier survey waves.

7. The difference-in-differences (DID), or baseline-adjusted treatment-control quasiexperimental modelling approach makes a different assumption to the LDV model: that the level of the outcome variable may differ between treatment and comparison 
groups, but the trends are parallel because unobserved differences do not vary over time. The use of this approach versus LDV is based on the plausibility of the underlying assumptions. We tested the robustness of our results to this alternate modelling approach, using kernel-based propensity score matching with the Epanechnikov kernel function and a probit propensity score model to create a control group with similar observable factors to the treatment group. The propensity scores were estimated using the full set of covariates included in the regression model. The model we estimated was a two-period, two-group model, so that MAT was compared to a pooled reference category of some or no treatment, and the difference between treatment and some or no treatment groups is compared at baseline and follow up.

8. The MAT variable categorizes number of mental health visits as fewer than four, four to 8 , and more than 8 visits. To examine the relationship between outcome and number of visits as a continuous variable, we graphically examined the association of change in total PEDSQL and number of mental health visits between baseline and follow-up, using a scatter plot and quadratic prediction plot.

\section{Results}

\section{$\underline{\text { Sample characteristics }}$}

The sample consisted of 596 children with SDQ of $\geq 17$ at baseline. Sample characteristics are shown in Table 1 . Less than half $(40 \%)$ of the sample had any mental health treatment recorded, and $12 \%$ received treatment classified as MAT.

[Insert Table 1 here] 
As shown in Table 1, unadjusted total PEDSQL and SDQ scores did not differ significantly between treatment groups at baseline, but the MAT group had lower psychosocial quality of life at baseline, and higher internalizing problem scores on SDQ. The pattern of mean PEDSQL scores over time varied slightly between the three treatment categories, as seen in Figure 2, with all three groups showing a decline from pre-baseline to baseline, and then improvement at follow-up. At every time point the mean PEDSQL in our sample was substantially below the LSAC mean. A similar pattern is seen for SDQ scores in Figure 3.

[Insert Figure 2 here]

Figure 2. Mean PEDSQL scores (total and psychosocial) over time by treatment category. [Insert Figure 3 here]

Figure 3. Mean SDQ scores (total, internalizing, and externalizing) over time by treatment category

\section{$\underline{\text { Regression results }}$}

Results of the LDV regression modelling in Figure 4 (and TableS2) show no statistically significant association between MAT treatment status and quality of life at follow-up either measured on total PEDSQL (point estimate 1.2, 95\% CI -2.2 to 4.6) or the psychosocial scale of the PEDSQL (point estimate $0.45,95 \%$ CI -3.3 to 4.2 ). There was a positive association between quality of life within individuals across time points. The other explanatory variables did not show a statistically significant association with quality of life apart from a negative association with parental depressive symptoms in the year prior to baseline for the psychosocial scale of the 
PEDSQL. To test whether collinearity between the treatment variables and covariates was contributing to the results, we excluded the covariates from the model, and there remained no significant association between MAT and outcomes.

[Insert Figure 4 here]

Figure 4. Association of treatment and covariates with quality of life at follow-up.

\section{Robustness checks}

The use of alternative models and sample definitions produced similar results regarding treatment (shown in TableS3-TableS9) to those in the main analysis.

1. Treatment status showed no statistically significant association with SDQ internalizing or externalizing scales at follow-up.

2. Treatment also showed no statistically significant association with the subdomains of psychosocial quality of life at follow-up.

3. With MAT specified as an 'implemented GP mental health plan', the sample was larger $(\mathrm{N}=777)$ due to the inclusion of prior waves of the survey. The results were similar to the main analysis, with no association between treatment status and quality of life.

4. Dropping pediatrician visits from the definition of mental health visits in MAT did not change the findings. 
5. Using a higher threshold on the SDQ for inclusion into the sample $(\mathrm{N}=414)$ displayed no association between MAT and quality of life.

6. Using the self-reported SDQ to define the sample generated a larger sample size $(\mathrm{N}=911)$ but the analysis on this sample also showed no statistically significant association between MAT and quality of life.

7. The DID model also found no statistically significant effect of treatment on quality of life.

8. As FigureS1 shows, there was no relationship between total number of visits as a continuous variable and change in PEDSQL from baseline to follow-up.

\section{Discussion}

This study examines an important question regarding the impact of treatment for children's mental health problems in real-world practice. It is to our knowledge the first to test whether treatment defined as 'minimally adequate' according to clinical practice guidelines is associated with improved outcomes for children with mental health problems. We found no statistically significant association between MAT and quality of life or mental health symptoms either in unadjusted analysis or when accounting for observed and unobservable differences between treatment groups. This finding is robust across a range of alternate specifications, although the confidence intervals around the point estimates include potentially substantial positive or negative associations. These results are in contrast to clinical trials which show that treatment can be efficacious for children with mental health problems, but are consistent with a limited pool of evidence in routine clinical practice showing less benefit of treatment (Weisz and Jensen, 2001; Weisz et al., 2013). 
Few other studies have examined the impact of MAT on outcomes. Among adults with depression, Duhoux et al. (2013) found that those who received MAT had larger improvements in depression symptoms. While that study measured severity and other important factors through primary data collection, it did not condition the findings on pre-treatment trends, and the modelling approach made the assumption that unobserved factors did not influence the likelihood of treatment. Fortney et al. (2001) addressed the problem of selection bias using an instrumental variable approach, and were able to differentiate severity based on clinical diagnosis. They found a larger benefit of guideline-concordant care for patients with major depression once selection bias was accounted for using the Heckman selection model.

There are several factors that may contribute our findings of no association between MAT and children's outcomes. Heterogeneity may obscure a true effect for some children who receive MAT. Different types of treatment were included in this definition of MAT, which allows for provision by different types of healthcare professionals, and different therapeutic approaches. These different types of treatment may differ in their impact on outcomes. There is also potential for heterogeneity in the clinical conditions included in our sample, since SDQ is a non-specific indicator of mental health problems, and we lack diagnostic data. Another possibility is variation in quality: the administrative data used in this study do not allow us to differentiate between high and lower quality care, or to detect the appropriateness of treatment for the clinical condition. Routine care may differ in quality from that provided within the clinical trial context, or may be variable in quality, leading to a lack of impact on outcomes at the aggregate level.

We have focused on generic outcome variables (quality of life and SDQ), which are able to discriminate between clinically distinct groups. However, there may be domains of benefit of MAT that are not captured by these measures. Disorder-specific outcome measures or clinician- 
rated outcome measures may be more sensitive to these changes. Providers of community-based mental health care in Australia are not required to report data on health outcomes. While the clinician may employ outcome measurement for their own use, there are no requirements for reporting of diagnoses, treatment type, quality, or outcomes of care. This limits the routine monitoring of treatment impact for most children receiving mental health care. The individual treating clinician is able to directly assess ongoing status of their patient, but lacks a counterfactual - how the patient would have progressed if a different treatment regime were followed. With the addition of diagnosis and outcome measures to routinely collected data, statistical analysis would be able to more fully answer the question of the whether MAT improves children's outcomes, and the funder of care would be able to monitor care quality and impact.

\section{Strengths and limitations}

A key strength of our study is its statistical approach, which addresses unobserved confounding by factors that are associated with both treatment and outcomes. The LDV regression method allows for different levels and trends of quality of life in the MAT and comparison groups. Children with and without treatment showed improvement in quality of life between baseline and follow-up. Improvement seen on repeated measurement may in part be due to regression to the mean, which reinforces the importance of including a comparison group susceptible to the same phenomenon, as we did. There were small but potentially important differences in pre-treatment trajectories between the treatment groups, and it was feasible that unobserved confounding factors (such as clinical severity) varied over time. The LDV model assumes that conditioning on past outcomes at multiple time points accounts for time-varying differences between treatment and comparison groups. The DID model makes a different assumption - that unobserved 
differences remain steady over time - but produced the same finding regarding the impact of treatment. An instrumental variable approach was not feasible in this study due to the absence of a viable instrument.

The use of nationally representative longitudinal individual data with a rich set of covariates on child and family characteristics, linked to detailed treatment data allowed us to control for individual characteristics before treatment.

The main limitations of the study relate to the comprehensiveness and accuracy of the data. MBS data do not capture tertiary community-based mental health services (Child and Adolescent Mental Health Services - CAMHS), fully privately funded consultations not billed to Medicare (the public insurer), hospital inpatient care, or school-based services. Identifying children receiving these other forms of treatment as untreated would lead to an underestimate of the treatment effect. However, entirely privately billed consultations are expected to be rare since few patients cannot be billed through Medicare and this does not restrict out-of-pocket payments. Additionally, recent Australian survey data showed that only 3\% of children with mental disorders received care in CAMHS and 6\% received mental health care in emergency departments, hospital outpatient clinics, or were admitted for mental health for mental health reasons (Lawrence et al., 2015). The lack of data on school-based counselling is a more important limitation, as the survey indicated that approximately $6 \%$ of children with mental disorders received ten or more sessions of individual counselling at school within twelve months, which may constitute MAT although little is known about the provider type or nature of the interventions offered (Lawrence et al., 2015). Future research with links to education services would be valuable. 
Additionally, inclusion in the sample was not based on clinical assessment and outcomes were not measured in terms of clinical recovery. The available variables in LSAC did not allow for the identification of different types of mental health disorders, which may have different requirements for and responses to treatment. However, the PEDSQL and SDQ are well-validated and widely used measures of quality of life and mental health in children, respectively (Goodman et al., 2000; Varni et al., 2003) and SDQ is used by mental health services to routinely assess patient outcomes (Burgess et al., 2015). The findings did not change when we used a higher SDQ threshold of 20 to identify a sample with higher probability of clinical diagnosis, nor with the sample defined using self-reported SDQ rather than parent reported. It would be valuable to replicate this study using a sample defined by clinical diagnosis and/or with a clinical outcome measure if such a population-level dataset were to become available with longitudinal follow up. We were not able to use self-reported outcomes in this study, but this could be an area for future studies as these measures may be more sensitive to the impact of treatment on emotional symptoms, or may pick up different important aspects of symptoms important to children.

\section{Conclusions}

We found no evidence of improvement in quality of life or mental health symptoms for children who received a theoretically 'minimally adequate' level of treatment compared to those who received lower levels of treatment. The lack of evidence for a relationship between MAT and outcomes in children has implications for how we evaluate and fund mental health systems. Less than half $(40 \%)$ of children with mental health symptoms in this sample received any treatment, consistent with a worldwide picture of inadequacies of children's mental health care (Rocha et al., 2015). It is less clear how to interpret the fact that only one third of those receiving treatment 
(12\% of the total sample) received MAT during a two-year period. This study suggests that while MAT may provide important insights into the distribution of care, we cannot infer differences in outcomes based on measurement of MAT in routinely collected data. This limits the evaluation of health system performance and highlights the need for a policy focus on more granular measurement of care and outcomes in mental health. The lack of information on quality or content of consultations, or on outcomes, in routinely collected health service data restricts our ability to evaluate treatment adequacy and to understand whether mental health services provide quality care or value for money. Our findings reinforce the need for routine monitoring of outcomes in mental health care (Trask and Garland, 2012) and better understanding of the impact of children's mental health treatment in routine practice (Weisz and Jensen, 2001). 
Tables

Table 1. Sample characteristics at baseline $(\mathrm{N}=596)$.

\begin{tabular}{|c|c|c|c|c|c|c|}
\hline & \multicolumn{3}{|c|}{$\begin{array}{l}\text { Treatment between baseline and follow up } \\
\text { (mental health visits and medication) }\end{array}$} & \multicolumn{3}{|c|}{ Tests of difference } \\
\hline & MAT & $\begin{array}{c}\text { Some } \\
\text { treatment }\end{array}$ & No treatment & $\begin{array}{l}\text { MAT vs. } \\
\text { Some vs. } \\
\text { No } \\
\text { treatment b }\end{array}$ & $\begin{array}{l}\text { MAT vs. } \\
\text { Some } \\
\text { treatment }^{\mathrm{c}}\end{array}$ & $\begin{array}{l}\text { MAT vs. } \\
\quad \text { No } \\
\text { treatment }^{\mathrm{c}}\end{array}$ \\
\hline \multirow[t]{2}{*}{$\mathrm{N}(\%$ of sample $)$} & $71(12 \%)$ & $165(28 \%)$ & $360(60 \%)$ & & & \\
\hline & $\% \quad$ Mean & $\% \quad$ Mean & $\% \quad$ Mean & $\mathrm{p}$ & $\mathrm{p}$ & $\mathrm{p}$ \\
\hline \multicolumn{7}{|l|}{ Child characteristics } \\
\hline $\begin{array}{l}\text { Parent report of diagnosed mental disorder at } \\
\text { any time prior to baseline }\end{array}$ & $57 \cdot 7$ & $49 \cdot 7$ & $43 \cdot 3$ & $0 \cdot 058$ & 0.256 & 026 \\
\hline Any mental health treatment prior to baseline & $84 \cdot 5$ & $66 \cdot 1$ & $22 \cdot 5$ & $<0 \cdot 001$ & 0.004 & $<0.001$ \\
\hline Female & $42 \cdot 2$ & $44 \cdot 2$ & $37 \cdot 8$ & $0 \cdot 346$ & 0.778 & 0.479 \\
\hline Age (years) & $10 \cdot 5$ & $10 \cdot 9$ & $10 \cdot 8$ & $0 \cdot 373$ & 0.172 & 0.343 \\
\hline $\begin{array}{l}\text { Indigeneity (Aboriginal or Torres Strait } \\
\text { Islander) }\end{array}$ & $2 \cdot 8$ & $4 \cdot 2$ & $3 \cdot 9$ & $0 \cdot 872$ & 0.600 & 0.662 \\
\hline
\end{tabular}




\begin{tabular}{|c|c|c|c|c|c|c|c|c|c|}
\hline \multicolumn{10}{|l|}{ Quality of life and mental health measures } \\
\hline PEDSQL total score & & $60 \cdot 4$ & & 60.2 & & $62 \cdot 5$ & $0 \cdot 156$ & 0.942 & 0.236 \\
\hline PEDSQL psychosocial scale & & $53 \cdot 6$ & & 54.9 & & $57 \cdot 4$ & $0 \cdot 026$ & 0.486 & 0.031 \\
\hline SDQ total score & & $20 \cdot 4$ & & 19.9 & & $20 \cdot 1$ & $0 \cdot 569$ & 0.288 & 0.392 \\
\hline SDQ internalizing scale & & $10 \cdot 2$ & & 9.3 & & $9 \cdot 2$ & $0 \cdot 018$ & 0.018 & 0.005 \\
\hline SDQ externalizing scale & & $10 \cdot 1$ & & 10.6 & & $10 \cdot 9$ & $0 \cdot 198$ & 0.248 & 0.076 \\
\hline \multicolumn{10}{|l|}{ Parent and family characteristics } \\
\hline Parent depression 2 weeks or more in last year & $74 \cdot 6$ & & $53 \cdot 9$ & & $43 \cdot 1$ & & $<0 \cdot 001$ & 0.003 & $<0.001$ \\
\hline Parent reports any mental illness at wave $5^{\text {a }}$ & $64 \cdot 8$ & & $53 \cdot 3$ & & $38 \cdot 9$ & & $<0 \cdot 001$ & 0.103 & $<0.001$ \\
\hline Parent K6 score & & $13 \cdot 5$ & & $11 \cdot 3$ & & $10 \cdot 6$ & $<0 \cdot 001$ & $<0.001$ & $<0.001$ \\
\hline Mother more than high school education & $83 \cdot 1$ & & $83 \cdot 6$ & & $75 \cdot 6$ & & $0 \cdot 069$ & 0.919 & 0.169 \\
\hline Remote area of residence & $1 \cdot 4$ & & $3 \cdot 0$ & & $5 \cdot 3$ & & $0 \cdot 225$ & 0.468 & 0.157 \\
\hline Language other than English spoken at home & $5 \cdot 6$ & & $7 \cdot 3$ & & $6 \cdot 4$ & & $0 \cdot 881$ & 0.646 & 0.810 \\
\hline Weekly household income (\$AU) & & 2,532 & & 2,488 & & 2,443 & $0 \cdot 909$ & 0.873 & 0.699 \\
\hline \multicolumn{10}{|l|}{ Treatment measures } \\
\hline $\begin{array}{l}\text { GP mental health plan } \& \geq 6 \text { mental health visits } \\
\text { between baseline and follow up }\end{array}$ & $43 \cdot 7$ & & $0 \cdot 9$ & & $\mathrm{n} / \mathrm{a}$ & & & & \\
\hline
\end{tabular}

${ }^{a}$ Only reported at wave 5 , not at baseline for all 
${ }^{\mathrm{b}}$ One-way analysis of variance test for continuous variables, chi-square test for binary variables.

${ }^{\mathrm{C}}$ Two-sample t-test for continuous variables, chi-square test for binary variables

Treatment between baseline and follow up:

- $\mathrm{MAT}=$ meets criteria for MAT

- Some treatment=at least one mental health visit or medication but not sufficient for MAT

- No treatment=no mental health visits or medication 


\section{References}

Adamo, N., Seth, S., Coghill, D., 2015. Pharmacological treatment of attentiondeficit/hyperactivity disorder: assessing outcomes. Expert review of clinical pharmacology 8 , 383-397.

Burgess, P., Pirkis, J., Coombs, T., 2015. Routine outcome measurement in Australia. International Review of Psychiatry 27, 264-275.

Coghill, D., Danckaerts, M., Sonuga-Barke, E., Sergeant, J., Group, A.E.G., 2009. Practitioner review: quality of life in child mental health-conceptual challenges and practical choices.

Journal of Child Psychology and Psychiatry 50, 544-561.

Cully, J.A., Zimmer, M., Khan, M.M., Petersen, L.A., 2008. Quality of depression care and its impact on health service use and mortality among veterans. Psychiatric Services 59, 1399-1405. Dewa, C.S., Hoch, J.S., Lin, E., Paterson, M., Goering, P., 2003. Pattern of antidepressant use and duration of depression-related absence from work. British Journal of Psychiatry 183, 507513.

Duhoux, A., Fournier, L., Gauvin, L., Roberge, P., 2013. What is the association between quality of treatment for depression and patient outcomes? A cohort study of adults consulting in primary care. Journal of Affective Disorders 151, 265-274.

Fortney, J., Rost, K., Zhang, M., Pyne, J., 2001. The relationship between quality and outcomes in routine depression care. Psychiatric Services 52, 56-62.

Goodman, A., Lamping, D.L., Ploubidis, G.B., 2010. When to Use Broader Internalising and Externalising Subscales Instead of the Hypothesised Five Subscales on the Strengths and 
Difficulties Questionnaire (SDQ): Data from British Parents, Teachers and Children. Journal of Abnormal Child Psychology 38, 1179-1191.

Goodman, R., Ford, T., Simmons, H., Gatward, R., Meltzer, H., 2000. Using the Strengths and Difficulties Questionnaire (SDQ) to screen for child psychiatric disorders in a community sample. British Journal of Psychiatry 177, 534-539.

Gray, M., Sanson, A., 2005. Growing up in Australia: The longitudinal study of Australian children. Family Matters, 4.

Hahm, H.C., Cook, B.L., Ault-Brutus, A., Alegría, M., 2015. Intersection of race-ethnicity and gender in depression care: screening, access, and minimally adequate treatment. Psychiatric Services 66, 258-264.

Hawes, D.J., Dadds, M.R., 2004. Australian data and psychometric properties of the Strengths and Difficulties Questionnaire. Australian and New Zealand Journal of Psychiatry 38, 644-651. Jorm, A.F., Patten, S.B., Brugha, T.S., Mojtabai, R., 2017. Has increased provision of treatment reduced the prevalence of common mental disorders? Review of the evidence from four countries. World Psychiatry 16, 90-99.

Kataoka, S., Zhang, L., Wells, K., 2002. Unmet Need for Mental Health Care Among U.S. Children: Variation by Ethnicity and Insurance Status. American Journal of Psychiatry 159, 1548-1555.

Lawrence, D., Johnson, S., Hafekost, J., De Haan, K.B., Sawyer, M., Ainley, J., Zubrick, S.R., 2015. The mental health of children and adolescents. Report on the second Australian Child and Adolescent Survey of Mental Health and Wellbeing. Canberra: Department of Health. MBS Online, GP mental health treatment items (Items 2700 to 2717) Department of Health. 
Mellor, D., 2005. Normative data for the strengths and difficulties questionnaire in Australia. Australian Psychologist 40, 215-222.

Merikangas, K.R., He, J.-p., Burstein, M., Swendsen, J., Avenevoli, S., Case, B., Georgiades, K., Heaton, L., Swanson, S., Olfson, M., 2011. Service Utilization for Lifetime Mental Disorders in U.S. Adolescents: Results of the National Comorbidity Survey-Adolescent Supplement (NCSA). Journal of the American Academy of Child \& Adolescent Psychiatry 50, 32-45.

O’Neill, S., Kreif, N., Grieve, R., Sutton, M., Sekhon, J.S., 2016. Estimating causal effects: considering three alternatives to difference-in-differences estimation. Health Services and Outcomes Research Methodology 16, 1-21.

Polanczyk, G.V., Salum, G.A., Sugaya, L.S., Caye, A., Rohde, L.A., 2015. Annual Research Review: A meta-analysis of the worldwide prevalence of mental disorders in children and adolescents. Journal of Child Psychology and Psychiatry 56, 345-365.

Puyat, J.H., Kazanjian, A., Goldner, E.M., Wong, H., 2016. How often do individuals with major depression receive minimally adequate treatment? A population-based, data linkage study. The Canadian Journal of Psychiatry 61, 394-404.

Rocha, T.B.-M., Graeff-Martins, A.S., Kieling, C., Rohde, L.A., 2015. Provision of mental healthcare for children and adolescents: a worldwide view. Current opinion in psychiatry 28, $330-335$.

Saloner, B., Carson, N., Cook, B.L., 2014. Episodes of mental health treatment among a nationally representative sample of children and adolescents. Medical Care Research and Review 71, 261-279. 
Sawyer, M.G., Reece, C.E., Sawyer, A.C., Hiscock, H., Lawrence, D., 2018a. Adequacy of treatment for child and adolescent mental disorders in Australia: A national study. Australian \& New Zealand Journal of Psychiatry, 0004867418808895.

Sawyer, M.G., Reece, C.E., Sawyer, A.C., Johnson, S.E., Lawrence, D., 2018b. Has the Prevalence of Child and Adolescent Mental Disorders in Australia Changed Between 1998 and 2013 to 2014? Journal of the American Academy of Child \& Adolescent Psychiatry 57, 343-350. e345.

Smith, E.G., Henry, A.D., Zhang, J., Hooven, F., Banks, S.M., 2009. Antidepressant Adequacy and Work Status Among Medicaid Enrollees with Disabilities: A Restriction-based, Propensity Score-adjusted Analysis. Community Mental Health Journal 45, 333-340.

Thornicroft, G., Chatterji, S., Evans-Lacko, S., Gruber, M., Sampson, N., Aguilar-Gaxiola, S., Al-Hamzawi, A., Alonso, J., Andrade, L., Borges, G., 2017. Undertreatment of people with major depressive disorder in 21 countries. The British Journal of Psychiatry 210, 119-124. Trask, E.V., Garland, A.F., 2012. Are Children Improving? Results from Outcome Measurement in a Large Mental Health System. Administration and Policy in Mental Health and Mental Health Services Research 39, 210-220.

Varni, J.W., Burwinkle, T.M., Seid, M., Skarr, D., 2003. The PedsQL ${ }^{\text {TM* }} 4.0$ as a Pediatric Population Health Measure: Feasibility, Reliability, and Validity. Ambulatory Pediatrics 3, 329341.

Wang, P.S., Lane, M., Olfson, M., Pincus, H.A., Wells, K.B., Kessler, R.C., 2005. Twelvemonth use of mental health services in the United States: results from the National Comorbidity Survey Replication. Archives of general psychiatry 62, 629-640. 
Weisz, J.R., Jensen, A.L., 2001. Child and adolescent psychotherapy in research and practice contexts: Review of the evidence and suggestions for improving the field. European Child \& Adolescent Psychiatry 10, S12.

Weisz, J.R., Ugueto, A.M., Cheron, D.M., Herren, J., 2013. Evidence-based youth psychotherapy in the mental health ecosystem. Journal of Clinical Child \& Adolescent Psychology 42, 274-286.

Youth in Mind, 2016. Information for researchers and professionals about the Strengths \& Difficulties Questionnaires. Youth in Mind 


\section{University Library}

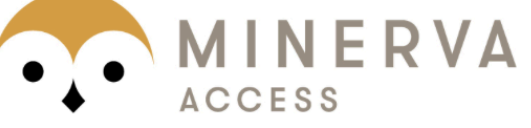

A gateway to Melbourne's research publications

Minerva Access is the Institutional Repository of The University of Melbourne

\section{Author/s:}

Ride, J;Huang, L;Mulraney, M;Hiscock, H;Coghill, D;Sawyer, M;Sciberras, E;Dalziel, K

Title:

Is 'minimally adequate treatment' really adequate? investigating the effect of mental health treatment on quality of life for children with mental health problems

Date:

2020-11-01

\section{Citation:}

Ride, J., Huang, L., Mulraney, M., Hiscock, H., Coghill, D., Sawyer, M., Sciberras, E. \& Dalziel, K. (2020). Is 'minimally adequate treatment' really adequate? investigating the effect of mental health treatment on quality of life for children with mental health problems. JOURNAL OF AFFECTIVE DISORDERS, 276, pp.327-334. https://doi.org/10.1016/ j.jad.2020.07.086.

Persistent Link:

http://hdl.handle.net/11343/241480 